\title{
Perfil das Pesquisas em Contabilidade de Custos Apresentadas no EnANPAD no Período de 1998 a 2003
}

\author{
Ricardo Lopes Cardoso \\ Carlos Alberto Pereira \\ Reinaldo Guerreiro
}

\section{RESUMO}

As pesquisas em contabilidade têm sido marcadas por novas abordagens para gestão de custos nas organizações e por estudos multidisciplinares de certos fenômenos sob diferentes perspectivas. $\mathrm{O}$ objetivo deste artigo é traçar um perfil da pesquisa em custos no âmbito da temática de contabilidade e controle gerencial do ENANPAD, desde a sua inauguração como área autônoma em 1998 até 2003. A análise consistiu inicialmente na revisão de 170 artigos aceitos na área temática e, depois, na seleção dos 32 artigos específicos de custos. Foram pesquisados os temas abordados, os métodos de pesquisa adotados, os segmentos econômicos estudados, a filiação acadêmica dos autores e o tipo de bibliografia consultada. Os resultados evidenciaram dois principais fatores: (i) um forte crescimento quantitativo da temática de contabilidade, passando do patamar de 40 trabalhos submetidos em 1998 para 191 trabalhos em 2003, com reflexos quantitativos relevantes na área de custos; e (ii) que este crescimento quantitativo não foi acompanhado, na mesma proporção, pelo crescimento qualitativo dos trabalhos na área de custos. A maior contribuição deste artigo é retratar o estado da arte da pesquisa em contabilidade de custos em evento científico brasileiro de alta qualidade.

Palavras-chave: pesquisa em contabilidade; contabilidade de custos e controle gerencial.

\begin{abstract}
Accounting researches have been marked by new approaches in order to manage costs in organizations and by multidisciplinary studies about certain phenomena from different perspectives. This article aims to draw up a profile of cost research in the framework of the accounting and management control theme area during the meetings of the Brazilian National Association of Graduate Programs in Business Administration (ENANPAD) since its inauguration as an autonomous area in 1998 until 2003. Initially, our analysis involved the review of 170 articles accepted in this theme area. Afterwards, we selected 32 specific articles on cost accounting. We examined the analyzed themes, the adopted research methods, which economic segments were investigated, the authors' academic affiliation and the kind of bibliography they had consulted. The results evidenced two main factors: (i) a strong quantitative growth in the accounting theme, increasing from 40 submitted papers in 1998 to 191 in 2003, with considerable quantitative influence in the cost area; and (ii) this quantitative growth was not accompanied, to the same extent, by the qualitative progress of papers in the cost area. This article presents the state-of-the-art of cost accounting research at a Brazilian high-quality scientific event.
\end{abstract}

Key words: research in accounting; cost accounting and management control. 


\section{INTRODUÇÃO}

O ambiente econômico e social tem-se modificado profundamente nas últimas décadas, levando muitas empresas a adotarem novos modelos de gestão empresarial e, conseqüentemente, a novas demandas por informações. A contabilidade tem o papel fundamental de suprir as necessidades de informações para a gestão empresarial. Com esse propósito, têm surgido, no campo teórico e prático da contabilidade, novas abordagens para a gestão de custos nas organizações, tais como: ABC - Activity Based Costing, ABM - Activity Based Management, BSC - Balanced Scorecard, GECON - Gestão Econômica, dentre outras. Algumas correntes do pensamento contábil têm se utilizado de outros ramos do conhecimento - como a psicologia e a sociologia - visando ao entendimento de certos fenômenos observados na prática contábil. A multidisciplinaridade começa a fazer parte das pesquisas na área contábil, dando origem ao estudo de temas emergentes em contabilidade, envolvendo teorias oriundas de outros ramos do conhecimento. A teoria institucional, por exemplo, é uma abordagem sociológica que tem sido adotada por diversos pesquisadores na área contábil, para entender os fenômenos da estabilidade e da mudança em contabilidade gerencial (Burns, 2000; Burns \& Scapens, 2000; Scapens, 1994; Scapens \& Roberts, 1993).

No Brasil, observa-se uma intensificação da pesquisa na área contábil, impulsionada principalmente pelos seguintes fatores: (i) surgimento de vários programas de pós-graduação na área de contabilidade; (ii) aumento das exigências da CAPES - órgão governamental responsável pelos programas de pós-graduação no país - com relação ao reconhecimento e avaliação desses programas; (iii) aumento da quantidade de eventos e periódicos científicos.

Nesse contexto, surgem os seguintes questionamentos: Como tem evoluído a pesquisa em contabilidade de custos no Brasil? A evolução quantitativa dos trabalhos tem sido acompanhada pelo aumento de qualidade das pesquisas realizadas? Os trabalhos desenvolvidos têm sido mais de natureza teórica ou empírica? Quais foram os temas estudados, os métodos de pesquisa adotados, os segmentos econômicos selecionados e os tipos de bibliografia consultada? A busca de respostas a essas questões passa necessariamente pela escolha e análise de um universo de trabalhos científicos representativos da pesquisa em custos no Brasil.

O objetivo deste trabalho é traçar um perfil da pesquisa em custos no âmbito do EnANPAD no período de 1998 a 2003, ou seja, desde o surgimento da temática 
de Contabilidade e Controle Gerencial. O evento EnANPAD foi selecionado em função da sua qualidade reconhecida no meio científico brasileiro. Foram identificados os temas abordados, os métodos de pesquisa adotados, os segmentos econômicos estudados, a filiação acadêmica dos pesquisadores e a bibliografia referenciada nos artigos apresentados. Estudos deste tipo não são novos; contudo, na área de contabilidade e controle gerencial do EnANPAD, diferentemente de outras áreas, não se observam registros de trabalhos desta natureza.

A importância deste estudo é contribuir para a evidenciação da evolução quantitativa e qualitativa da pesquisa em contabilidade de custos no Brasil. Estudos desta natureza estão diretamente relacionados com a carreira dos docentes e com o desenvolvimento dos programas de pós-graduação em contabilidade. Ao traçar um panorama sobre o perfil da pesquisa em custos no âmbito do EnANPAD, pretende-se contribuir com a discussão sobre os rumos da produção científica em contabilidade no Brasil.

A apresentação e discussão dos resultados de pesquisas científicas em eventos como o EnANPAD constituem etapa importante do processo de produção do conhecimento científico. Vale ressaltar, contudo, que a carência de estudos sobre a evolução e o perfil da pesquisa em custos no Brasil dificulta uma discussão mais profunda sobre as contribuições dessas pesquisas para a evolução da teoria e a efetiva mudança da realidade prática da contabilidade, conforme discutem Sue (1996) e Ittner e Larcker (2002). Neste sentido, este trabalho gera alguns insights para uma discussão com maior embasamento sobre os rumos da produção científica em contabilidade de custos no Brasil.

\section{Referencial Teórico}

No âmbito nacional, identificam-se estudos semelhantes ao desenvolvido neste trabalho na área de finanças, na área de teoria das organizações e na área de recursos humanos. No campo de finanças, Leal, Oliveira, e Soluri (2003) desenvolveram estudo, utilizando amplo banco de dados de eventos EnANPAD, bem como os principais periódicos da área de finanças. Nas áreas de estudos das organizações e de recursos humanos, destaca-se o trabalho pioneiro de Machado da Silva, Cunha e Amboni (1990), que discutiram a produção acadêmica em administração, bem como o estudo de Vergara e Carvalho (1995) e mais recentemente o trabalho de Tonelli, Caldas, Lacombe e Tinoco (2003). Na área de marketing, o trabalho de Vieira (1998) levanta a produção científica e avalia os impactos das citações. Na área de contabilidade, destacam-se os trabalhos de Riccio, Sakata e Carastan (1999) e Riccio, Sakata e Segura (2001) referentes à 
pesquisa em contabilidade e os trabalhos de Martins (2002) e de Oliveira (2002) sobre a evolução da pesquisa em contabilidade. No âmbito internacional, existem vários estudos relacionados ao campo da administração, como o de Mitenko e Diamond (1994), que analisam vários periódicos e as dimensões das pesquisas publicadas internacionalmente.

No campo de pesquisa em contabilidade, Horngren (1989) apresenta sua reflexão a respeito das mudanças na contabilidade de custos, considerando o período de 32 anos de sua atividade como professor universitário. À parte de suas reminiscências pessoais, o autor apresenta pesquisa sobre evidências da mudança de ênfase nos manuais de contabilidade de custos, considerando o período de 1945 a 1970. Nesse período, podem-se observar o declínio da ênfase no assunto avaliação de estoques, a permanência de foco sobre o controle de custos e o aumento de ênfase no tópico tomada de decisão gerencial.

Vollmers (1996) desenvolveu pesquisa com base em manuais publicados sobre contabilidade de custos no período de 1920 até 1950 nos Estados Unidos da América. Essa pesquisa teve como objetivo refutar a tese, proposta por Johnson e Kaplan (1987), de que a contabilidade de custos perdeu sua relevância a partir de 1920 , tendo em vista que os acadêmicos passaram a dar mais importância à contabilidade financeira, e de que nada de novo em contabilidade de custos surgiu a partir da década de vinte do século passado.

Shields (1997) apresenta um estudo muito amplo da pesquisa em contabilidade gerencial, a partir da análise de 152 artigos publicados por pesquisadores americanos em seis grandes periódicos internacionais. $\mathrm{O}$ autor relata que os artigos estudados apresentam uma variedade de métodos de pesquisa e que os métodos utilizados com maior freqüência foram o método analítico, a pesquisa do tipo survey, a pesquisa de arquivos e a experimentação em laboratório.

Atkinson, Balakrishnan, Booth, Cote, Groot et al. (1997) desenvolveram um estudo expandindo o conteúdo do relatório produzido pelo Comitê de Pesquisa da Seção de Contabilidade Gerencial, publicado em 1995, que apresenta uma lista de tópicos de contabilidade gerencial que potencialmente deveriam ser pesquisados a partir da década de 90 . Três macrotópicos foram escolhidos como questões fundamentais para pesquisas futuras em contabilidade gerencial: (i) o papel da contabilidade gerencial nas mudanças organizacionais; (ii) a interação de contabilidade e estrutura organizacional; e (iii) o papel da informação contábil no apoio à tomada de decisão.

Os trabalhos de Bricker (1989), Keating (1995) e Zeff (1996) discutem os rumos da pesquisa em contabilidade, baseados em amplos levantamentos nos principais periódicos do mundo. Mais recentemente, destaca-se o estudo de 
Bhimani (2002) sobre as abordagens metodológicas apresentadas na pesquisa européia, com destaque para aquela produzida no European Accounting Review. O estudo de Otchere (2003) trata da análise evolutiva da pesquisa em contabilidade e finanças e o trabalho de Model (2005) enfatiza o uso dos estudos de casos em pesquisa de contabilidade gerencial e custos.

O trabalho de Baxter e Chua (2003) coloca o foco no distanciamento entre a pesquisa de contabilidade gerencial e as contribuições teóricas para o desenvolvimento deste ramo do conhecimento. Para os autores, a principal causa desse distanciamento é a falta de maior embasamento teórico e de desenhos metodológicos mais rigorosos e ousados. Posição semelhante foi apresentada anteriormente por Kasanen e Lukka (1995), que ressaltaram a questão da falta de embasamento e de instrumentos adequados nas pesquisas em contabilidade para possibilitar generalizações.

\section{Universo Pesquisado}

O EnANPAD surgiu em 1976 e atualmente se constitui no maior e mais qualificado evento, de acordo com o sistema Qualis (Capes), tendo grande expressão no campo da administração e da contabilidade. A edição de 2003, por exemplo, recebeu 2.332 trabalhos, dos quais 630 foram aceitos, o que correspondeu a um percentual de $27 \%$, conforme se observa na Tabela 1.

Efetuando-se uma comparação com parâmetros internacionais, pode-se considerar que o benchmarking natural do EnANPAD é o evento Academy of Management (AOM) que, apesar das diferenças citadas em alguns estudos nacionais, como o de Quintella (2003) e internacionais como o de Beatty (2002), se constitui num referencial interessante para se efetuar algumas comparações. No aspecto de crescimento, a quantidade de trabalhos submetidos ao $A O M$ em 2003 , por exemplo, teve um aumento de $20 \%$ em relação a 2002 , enquanto o EnANPAD apresentou um crescimento de $28 \%$ no mesmo período. Sem dúvida, trata-se do maior evento dentro da grande área de conhecimento da administração e não pode ser desprezado em termos comparativos.

O universo pesquisado neste trabalho refere-se aos artigos apresentados na temática de contabilidade e controle gerencial e, dentro dessa temática, na área de custos. O período pesquisado foi desde 1998, início da existência da área temática, até 2003. O volume de trabalhos submetidos na área temática totalizou 548 artigos, dos quais 170 foram aceitos, sendo estes últimos o foco deste estudo. A evolução da quantidade de submissões e de trabalhos aceitos no EnANPAD, 
com destaque para a área de contabilidade e controle gerencial e, dentro desta, para a área de custos, é apresentada na Tabela 1:

\section{Tabela 1: Evolução do Volume de Trabalhos Submetidos e Aceitos}

\begin{tabular}{ccccccccc}
\hline \multirow{2}{*}{ Ano } & \multicolumn{2}{c}{ Total Geral - EnANPAD } & \multicolumn{2}{c}{ Área Temática - CCG } & \multicolumn{2}{c}{ Custos } \\
\cline { 2 - 8 } & Submetidos & Aceitos & $\%$ & Submetidos & Aceitos & $\%$ & Aceitos & $\%$ \\
\hline 1998 & 807 & 250 & $31,0 \%$ & 40 & 12 & $30,0 \%$ & 1 & $8,3 \%$ \\
1999 & 893 & 270 & $30,2 \%$ & 35 & 11 & $31,4 \%$ & 1 & $9,1 \%$ \\
2000 & 1.300 & 363 & $27,9 \%$ & 85 & 21 & $24,7 \%$ & 5 & $23,8 \%$ \\
2001 & 1.385 & 418 & $30,2 \%$ & 70 & 23 & $32,9 \%$ & 10 & $43,5 \%$ \\
2002 & 1.822 & 551 & $30,2 \%$ & 127 & 47 & $37,0 \%$ & 9 & $19,1 \%$ \\
2003 & 2.332 & 630 & $27,0 \%$ & 191 & 56 & $29,3 \%$ & 6 & $10,7 \%$ \\
\hline Total & 8.539 & 2.482 & $29,1 \%$ & 548 & 170 & $31,0 \%$ & 32 & $18,8 \%$ \\
\hline
\end{tabular}

A importância e o crescimento da área de contabilidade e controle gerencial no EnANPAD podem ser constatados ao se verificar que, em 1999, a área representava 4,07\% do total de trabalhos apresentados no evento e, em 2003, este percentual chegou a $8,89 \%$, conforme se evidencia na Tabela 2:

\section{Tabela 2: Evolução da Participação de Trabalhos Aceitos na Área Temática e de Custos em Relação ao Total}

\begin{tabular}{c|c|c}
\hline Ano & Área temática - CCG em relação ao total & Área de Custos em relação ao total \\
\hline 1998 & $4,80 \%$ & $0,40 \%$ \\
\hline 1999 & $4,07 \%$ & $0,37 \%$ \\
\hline 2000 & $5,79 \%$ & $1,38 \%$ \\
\hline 2001 & $5,50 \%$ & $2,39 \%$ \\
\hline 2002 & $8,53 \%$ & $1,63 \%$ \\
\hline 2003 & $8,89 \%$ & $0,95 \%$ \\
\hline Total & $6,85 \%$ & $1,29 \%$ \\
\hline
\end{tabular}

Apesar do visível aumento da participação de trabalhos da área temática de contabilidade e controle gerencial no total de trabalhos aceitos, não se observa o 
mesmo comportamento dos trabalhos de custos em relação a esse mesmo total. Isso pode refletir o aumento do interesse dos pesquisadores por temas não especificamente relacionados a custos. Não se pode afirmar, contudo, que a queda observada no volume (Tabela 1) e na participação (Tabela 2) dos trabalhos aceitos na área de custos em relação ao total de trabalhos aceitos seja produto de uma diminuição do interesse dos pesquisadores por trabalhos relacionados a custos, uma vez que não existem evidências claras das razões que motivaram esse decréscimo. Essa redução pode ter sido causada, dentre outras variáveis, tanto pela superioridade na avaliação dos trabalhos relacionados a outros temas dentro da área temática, quanto por uma eventual diminuição do volume de trabalhos de custos submetidos ao evento, variáveis essas que não foram objeto de investigação desta pesquisa.

Outro ponto que evidencia a evolução da área de contabilidade é apresentado no gráfico a seguir e está relacionado com o percentual de aceitação, que fica, na média do período, com $31 \%$ contra $28,8 \%$ do evento como um todo. Também é possível notar o expressivo crescimento do número de submissões dentro da área, passando de 40 em 1998 para 191 em 2003, representando um crescimento de $480 \%$ em 6 anos contra $357 \%$ do evento EnANPAD como um todo.

Analisando-se o Gráfico 1, percebe-se um movimento mais acentuado dos percentuais de aceitação de trabalhos na temática de contabilidade e controle gerencial do que no EnANPAD como um todo:

\section{Gráfico 1: Evolução dos Percentuais de Aceitação}

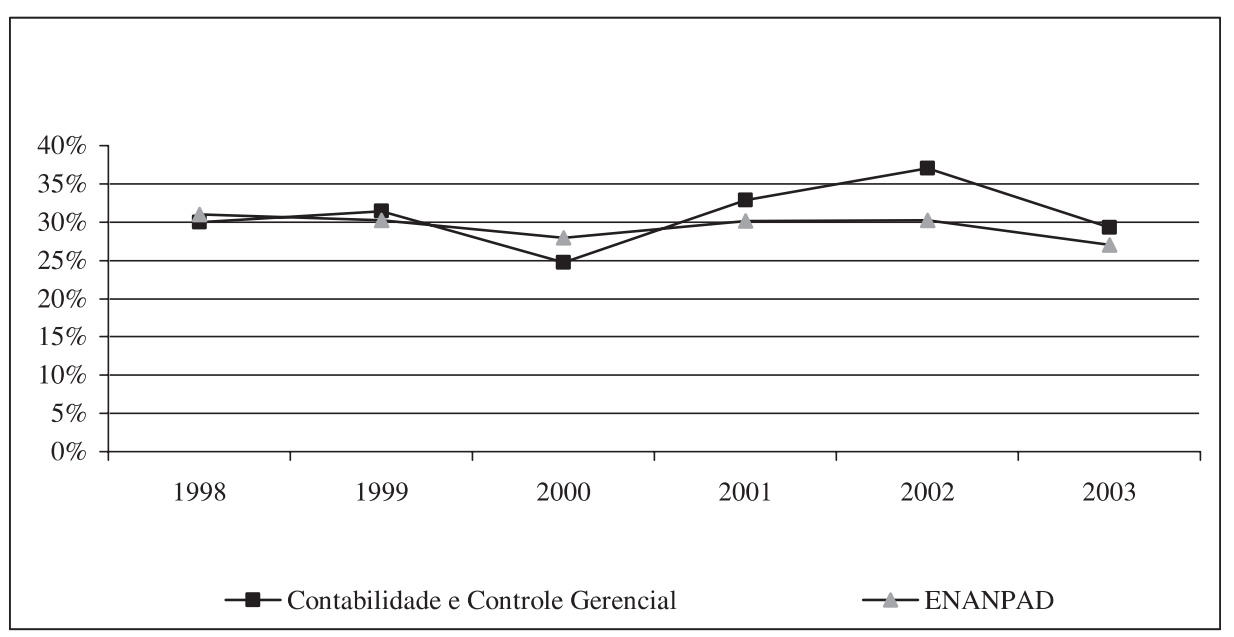




\section{Metodologia de Pesquisa}

Este artigo orienta-se pelas seguintes questões de pesquisa: (i) Como foi desenvolvida a pesquisa de custos no âmbito do EnANPAD até recentemente? (ii) Quais foram os principais temas explorados? (iii) Quais foram os métodos de pesquisa predominantes? (iv) Qual o perfil das publicações segundo as instituições, as regiões e os pesquisadores que realizaram os trabalhos? (v) Qual o perfil das obras consultadas e referenciadas nos trabalhos? A presente pesquisa baseou-se no método de coleta e análise utilizado pelos principais meta-estudos apresentados e discutidos nas diversas áreas do EnANPAD como, por exemplo, em Vieira (1998), Machado da Silva et al. (1990), Tonelli et al. (2003), que realizaram, de forma ampla, análise esquemática de algumas áreas temáticas do evento e de periódicos nacionais.

Os levantamentos ocorreram da seguinte forma: (i) separação dos anais de 1998 a 2003; (ii) definição do campo de abrangência da contabilidade de custos; (iii) leitura dos resumos e a separação destes por tema; (iv) leitura do tópico de metodologia e coleta de dados de cada artigo; e (v) simultaneamente foi elaborado um catálogo com todos os dados levantados (ano, tema, método de coleta, setor, título, autores, instituição e bibliografia).

No campo da variável temática, a classificação dos artigos da amostra esbarra, naturalmente, em discussões sobre as fronteiras da área de custos com outros segmentos da contabilidade, engenharia, administração, economia, entre outros. Procurando evitar esta polêmica, foram utilizadas as classificações temáticas preponderantes do Journal of Cost - Analysis e do Congresso Internacional de Custos, de modo a considerar como trabalho relacionado com custos todos aqueles que poderiam ser enquadrados nas temáticas deste periódico e do congresso. Os resultados temáticos apresentados, portanto, devem ser afetados por esses tipos de classificações, bem como por eventuais aspectos subjetivos utilizados no desenvolvimento dos levantamentos e análises. É importante observar que, para casos específicos, foi efetuado maior detalhamento do que o apresentado pelas referências mencionadas, objetivando maior transparência.

\section{Apresentação e Análise dos Resultados do Estudo}

A seguir são apresentados, em forma gráfica, os resultados obtidos, acompanhados das explicações necessárias. 


\section{Evolução dos Trabalhos de Custos Apresentados na Área Temática}

Conforme pode ser observado no Gráfico 2, no ano de 2001, houve um pico na participação dos trabalhos apresentados na área de custos, alcançando o percentual de $43,5 \%$ do total de artigos da área temática:

\section{Gráfico 2: Trabalhos Aceitos na Área Temática (CCG) e na Área de Custos}

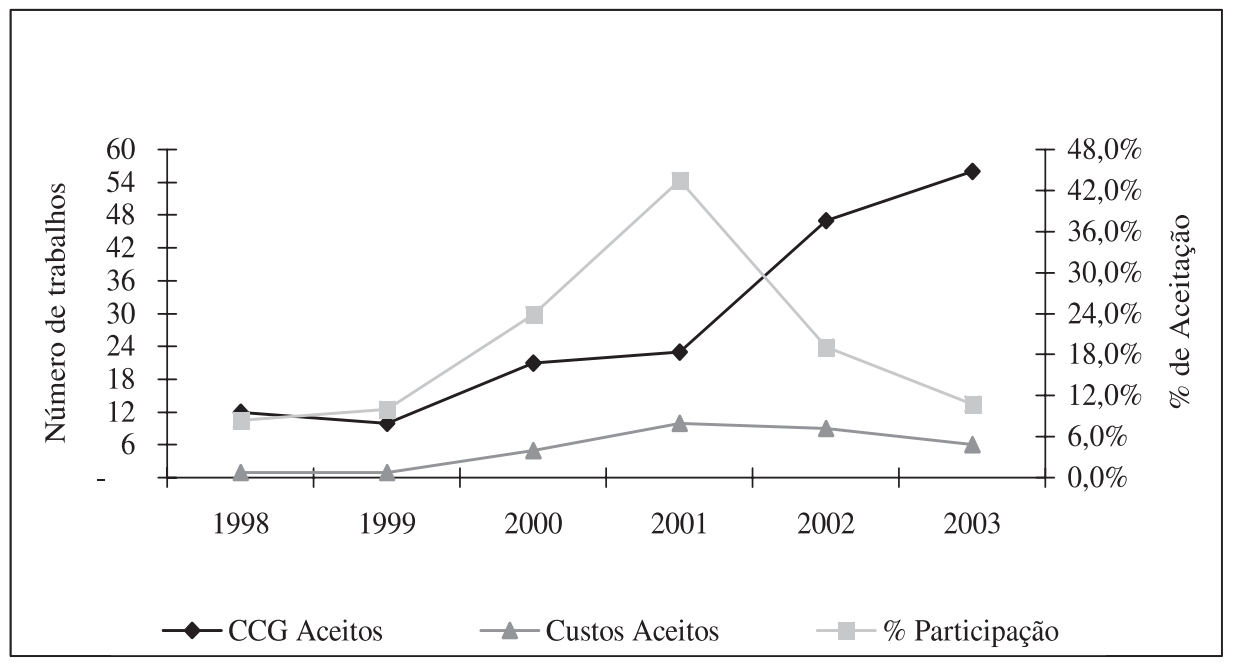

No período analisado, a participação média dos trabalhos apresentados na área de custos em relação ao total da área temática ficou em 19,8\%. Apesar do crescimento de trabalhos apresentados e da sua participação média, não se pode dizer que estes aumentos sejam constantes.

\section{Trabalhos de Custos por Tema}

Observando-se a Tabela 3, pode-se perceber claramente que 50\% dos 32 trabalhos apresentados sobre custos tratam do Sistema ABC e suas aplicações. Este fato demonstra a relevância deste tema de pesquisa contábil dentro do contexto nacional na época dos eventos. No estudo de Shields (1997), os temas relacionados com $\mathrm{ABC}$ na temática de contabilidade gerencial em 6 grandes periódicos internacionais representaram $10 \%$ do total de artigos publicados. Ainda com base na Tabela 3, percebe-se que, para os demais temas, a distribuição é pulverizada, podendo ser destacadas as pesquisas sobre 
os métodos de custeio utilizados pelas empresas, com 4 trabalhos diferentes, ou seja, $12,5 \%$ do total.

\section{Tabela 3: Composição Temática dos Trabalhos Apresentados}

\begin{tabular}{lcccccccc}
\hline \multicolumn{1}{c}{ Tópicos Classificados } & 1998 & 1999 & 2000 & 2001 & 2002 & 2003 & Total & $\%$ \\
\hline ABC & & & 3 & 4 & 6 & 3 & 16 & $50,0 \%$ \\
ABC x TOC & & & & & & 1 & 1 & $3,1 \%$ \\
Gecon & & & 1 & 1 & & 2 & $6,3 \%$ \\
Custo da qualidade e ambiental & & & & 1 & & & 1 & $3,1 \%$ \\
Custos nas empresas rurais & & & & 1 & & 1 & $3,1 \%$ \\
Preços & & 1 & 1 & & & 2 & $6,3 \%$ \\
Integração do Sistema de Custos & & & & 1 & & & 1 & $3,1 \%$ \\
Mix de Produtos & & & & 1 & & & 1 & $3,1 \%$ \\
Otimização da Produção & & & & & 1 & 2 & 4 & $12,5 \%$ \\
Sistemas de Custeio Usados & 1 & 1 & & & & & 1 & $3,1 \%$ \\
Ensino de custos & & & 1 & & & & 1 & $3,1 \%$ \\
Custos de projetos & & 1 & 5 & 10 & 9 & 6 & 32 & $100,0 \%$ \\
\hline Total & & & & & & &
\end{tabular}

\section{Publicação por Instituição Representada}

Apesar da existência de autores com filiações acadêmicas múltiplas, trabalhouse com o conceito de primeiro autor, para se contabilizar os trabalhos de acordo com a instituição à qual o autor está vinculado. Com este tratamento, nos casos dos artigos produzidos por alunos orientados em co-autoria com seus respectivos orientadores, a produção foi considerada como sendo da instituição do orientador. Para validar os casos de dúvidas, foram feitos acessos à base de dados do Currículo Lattes dos autores. De acordo com esses critérios, a distribuição das publicações por instituição representada no evento é apresentada na Tabela 4: 
Tabela 4: Publicações por Instituição Representada

\begin{tabular}{lc}
\hline \multicolumn{1}{c}{ Instituição } & Publicações \\
\hline UFPE & 5 \\
UFRJ & 5 \\
USP & 3 \\
PUC-SP & 3 \\
FECAP & 2 \\
Pedro Leopoldo & 2 \\
UFC & 1 \\
UFSC & 1 \\
UFF & 1 \\
UnB & 1 \\
UNIFOR & 1 \\
UENF & 1 \\
UFRP & 1 \\
UFPA & 1 \\
UFBA & 1 \\
UFSCar & 1 \\
UEM & 1 \\
Embrapa & 1 \\
\hline Total & 1 \\
\hline
\end{tabular}

Os resultados demonstraram que as instituições líderes em produção são a UFRJ e a UFPE, que apresentaram 5 trabalhos cada no período em análise. No caso da UFRJ, a participação maior está relacionada com as áreas de administração e engenharia. No caso da UFPE, quase a totalidade dos trabalhos está vinculada aos professores e alunos de mestrado em contabilidade. Em terceiro lugar, empatadas, aparecem a USP e a PUC-SP, participando com seus programas de pós-graduação em Ciências Contábeis. A participação de um número relativamente grande de instituições pode ser explicada pela criação recente de maior número de cursos de pós-graduação em contabilidade no Brasil. 


\section{Publicação por Região}

Refletindo a maior concentração dos programas de mestrado e mantendo o único doutorado em contabilidade no Brasil, o Estado de São Paulo publicou 29\% de todos os artigos, seguido pelo Rio de Janeiro com 22\%, Pernambuco com 19\% e Minas Gerais com 9\%, conforme dados apresentados na tabela 5. Estes dados também demonstram maior pulverização das áreas de custos e até mesmo da área de contabilidade. Este fator é estrutural e vem ocorrendo em todos os EnANPAD, sendo que, em 2001, 70\% da produção estava centralizada nas 11 primeiras instituições do ranking de publicações e, em 2002, este percentual caiu para 60\% (Quintella, 2003). Na área de custos do EnANPAD, não foi identificada nenhuma apresentação de trabalho de autoria internacional. Para fins ilustrativos é importante observar que o EnANPAD como um todo, na sua edição de 2002, recebeu 3\% de trabalhos estrangeiros para submissão e $4 \%$ do total de trabalhos apresentados foram do exterior. A AOM no mesmo período recebeu $30 \%$ de submissões estrangeiras.

\section{Tabela 5: Distribuição Geográfica dos Trabalhos de Custos}

\begin{tabular}{lc}
\hline \multicolumn{1}{r}{ Estado } & $\%$ \\
\hline Bahia & $3 \%$ \\
Ceará & $6 \%$ \\
Distrito Federal & $3 \%$ \\
Minas Gerais & $9 \%$ \\
Pará & $3 \%$ \\
Paraná & $3 \%$ \\
Pernambuco & $19 \%$ \\
Rio de Janeiro & $22 \%$ \\
Santa Catarina & $3 \%$ \\
São Paulo & $29 \%$ \\
\hline Total & $100 \%$ \\
\hline
\end{tabular}

\section{Publicação por Pesquisador}

A produção intelectual da temática de custos tem ocorrido, preponderantemente, através de parceria entre discentes e docentes, em linha com os critérios da 
Capes sobre o assunto. A apresentação de trabalhos de autoria individual correspondeu a somente $21,9 \%$ dos trabalhos de custos apresentados. A forma mais utilizada de parceria $(53,1 \%)$ correspondeu a trabalhos apresentados com dois autores, conforme evidencia a Tabela 6:

Tabela 6: Números de Autores por Artigo

\begin{tabular}{ccc}
\hline Número de Autores & Artigos & $\%$ \\
\hline 1 & 7 & $21,9 \%$ \\
2 & 17 & $53,1 \%$ \\
3 & 5 & $15,6 \%$ \\
4 & 3 & $9,4 \%$ \\
\hline Total & 32 & $100,0 \%$ \\
\hline
\end{tabular}

Uma visão complementar diz respeito à quantidade de artigos por autor que tem participado das publicações da área de custos. Dos 62 autores que constam da lista completa, apenas 5 apresentaram mais de um artigo no período de 1998 a 2003, conforme Tabela 7:

\section{Tabela 7: Número de Artigos por Autor}

\begin{tabular}{ccc}
\hline Autores & Número de Autores & $\%$ \\
\hline Com 1 artigo publicado & 57 & $92,0 \%$ \\
Com 2 artigos publicados & 3 & $4,8 \%$ \\
Com 3 artigos publicados & 2 & $3,2 \%$ \\
\hline Total & 62 & $100 \%$ \\
\hline
\end{tabular}

O grande número de pesquisadores com apenas um trabalho publicado na área de custos pode ter sido conseqüência de vários fatores, tais como: (i) o número expressivo de discentes que têm participado do evento; (ii) o período relativamente pequeno contemplado na pesquisa; (iii) os autores podem estar apresentando trabalhos em áreas afins de custo dentro da temática de contabilidade e controle gerencial; e, ainda, (iv) o problema do tempo de maturação das produções acadêmicas dos jovens mestres e doutores que ainda não submeteram mais trabalhos ao EnANPAD. A investigação dessas causas não foi objeto deste estudo, constituindo uma possível extensão desta pesquisa.

\section{Métodos de Coleta Utilizados nos Trabalhos Publicados}

Este foi o quesito de pesquisa de maior dificuldade para ser levantado e analisado. 
A estratégia utilizada foi detalhar os métodos de coleta de dados utilizados nos trabalhos publicados, sem a necessidade de enquadramento em nenhuma taxonomia geral predefinida. Foram definidos os seguintes métodos, para que fosse possível obter um retrato detalhado dos resultados:

. entrevistas, análise de dados e observação;

. modelos e aplicações;

. observação não estruturada;

. análise de textos;

. análise de textos e aplicação para validação;

. entrevistas e questionários; e

. entrevistas estruturadas.

Os resultados quanto aos métodos de coleta de dados utilizados nos trabalhos são apresentados na Tabela 8:

\section{Tabela 8: Métodos de Coleta de Dados}

\begin{tabular}{lc}
\hline \multicolumn{1}{c}{ Métodos } & $\%$ \\
\hline Modelos e aplicações & $27 \%$ \\
Entrevistas e questionários enviados & $22 \%$ \\
Análise de textos & $16 \%$ \\
Entrevistas, análise de dados e observação & $13 \%$ \\
Entrevistas estruturadas & $13 \%$ \\
Análise de textos e aplicação para validação & $6 \%$ \\
Observação não estruturada & $3 \%$ \\
\hline Total & $100 \%$ \\
\hline
\end{tabular}

Com base na Tabela 8, nota-se que alguns dos métodos de coleta (entrevistas e questionários enviados; entrevistas, análise de dados e observação; entrevistas estruturadas; análise de textos e aplicação para validação; observação não estruturada) evidenciam o caráter empírico dos artigos (57\%), embora se apresente grande volume de trabalhos fundamentados apenas em análise de textos (16\%) e modelos e aplicações (27\%), sem testes empíricos. No âmbito das entrevistas, foram segregados os dados de acordo com os 
tipos de entrevistas utilizados com a finalidade de demonstrar os detalhes metodológicos dos artigos.

Os estudos de casos não foram classificados e analisados em separado (28,1\% dos trabalhos utilizam esse método). Eisenhardt (1989) ressalta a importância e algumas limitações dos estudos de casos na construção de teorias. Nesse sentido, no campo da contabilidade gerencial, Otley e Berry (1994) observam que os estudos de casos têm potencialmente grande papel a desempenhar como instrumento de pesquisa, porém a partir de um enfoque exploratório e não meramente descritivo.

Os dados da amostra sugerem a falta de rigor na utilização desse método de pesquisa em relação ao que é proposto por alguns estudiosos do assunto (Yin, 2001). A falta de rigor na elaboração dos estudos de casos como meio de contribuição teórica é discutido em contabilidade gerencial inicialmente por Keating (1995) e por Model (2005), sendo que para esses autores parte da problemática está na falta do rigor metodológico utilizado e na falta da vinculação deste método a outras estratégias de pesquisas, objetivando a construção de caminhos mais sólidos para a contribuição teórica. A comparação entre a análise de Model (2005), realizada em âmbito internacional, e os dados relativos a estudos de casos desta pesquisa evidencia falta de rigor metodológico e explicitação das contribuições teóricas dos trabalhos. Essas considerações foram feitas a partir da análise dos procedimentos metodológicos utilizados nos artigos e das contribuições teóricas que não foram identificadas nos artigos pesquisados de forma explícita em termos de considerações finais.

Um aspecto relevante observado diz respeito ao método de pesquisa classificado como modelos de aplicação ( $27 \%$ dos trabalhos). Uma análise mais rigorosa indicaria que a quase totalidade desses trabalhos não se enquadraria em modelagem ou mesmo em criação de modelos, uma vez que não houve experimentação das propostas. É importante destacar que na área de custos não foi localizado nenhum artigo que tivesse utilizado pesquisa experimental para suas análises, o que não se alinha com a visão internacional colocada por Sprinkle (2003), em que são apresentadas evidências de uso deste tipo de pesquisa na área de contabilidade gerencial e mais especificamente em custos.

A análise dos dados evidencia, no entanto, que, nos últimos dois anos da pesquisa, houve maior adoção do enfoque empírico e maior preocupação com o tratamento metodológico. Problemas relativos ao método de pesquisa, como, por exemplo, a reduzida utilização do enfoque quantitativo e o pouco rigor na pesquisa qualitativa não parecem ser característica isolada da área de custos, uma vez que diversos autores (Machado-da-Silva et al., 1990; Quintella, 2003; Tonelli et al., 2003; Vergara \& Carvalho, 1995) já levantaram essa questão em outras áreas do EnANPAD. 


\section{Trabalhos por Segmento Econômico}

Com o objetivo de efetuar a classificação por segmento de atividade a que os trabalhos se referem, foram identificados 20 trabalhos de natureza aplicada diretamente a algum setor econômico e 12 trabalhos de natureza não aplicada (Tabela 9).

\section{Tabela 9: Aplicação a Setores Econômicos}

\begin{tabular}{lcc}
\hline \multicolumn{1}{c}{ Trabalhos } & Quantidade & $\%$ \\
\hline Aplicados & 20 & $62,5 \%$ \\
Não aplicados & 12 & $37,5 \%$ \\
\hline Total & 32 & $100 \%$ \\
\hline
\end{tabular}

A Tabela 10 demonstra a análise por setor de atividade dos 20 trabalhos que compõem os $62,5 \%$ da Tabela 9 . Como se pode observar, existe uma pulverização de setores envolvidos, sendo que $25 \%$ (geral) referem-se a pesquisas com aplicação de questionários amplos em diversos segmentos. O chamado agronegócio aparece em segundo lugar com 15\% dos trabalhos, seguido da área de saúde com 10\%.

Tabela 10: Trabalhos por Segmento Econômico

\begin{tabular}{|c|c|}
\hline Método & $\%$ \\
\hline Agronegócio & $15 \%$ \\
\hline Alimentos & $5 \%$ \\
\hline Automobilístico & $5 \%$ \\
\hline Geral & $25 \%$ \\
\hline Impressão & $5 \%$ \\
\hline Instituição de Ensino & $5 \%$ \\
\hline Metal & $5 \%$ \\
\hline Metalurgia & $5 \%$ \\
\hline Petróleo & $5 \%$ \\
\hline Saúde & $10 \%$ \\
\hline Seguros & $5 \%$ \\
\hline Turismo & $5 \%$ \\
\hline Varejo & $5 \%$ \\
\hline Total & $100 \%$ \\
\hline
\end{tabular}




\section{Classificação da Bibliografia}

Um ponto de destaque na análise qualitativa dos artigos publicados refere-se ao tipo de bibliografia referenciada. Para medir essa evolução, foi efetuado um estudo específico nas referências bibliográficas dos trabalhos. A bibliografia foi dividida inicialmente em nacional e internacional. Dentro de cada uma dessas classificações, a bibliografia foi segregada em (i) livros; (ii) teses e dissertações; (iii) periódicos; (iv) anais de eventos; (v) sites da web; (vi) jornais e magazines; (vii) normas; e (viii) outros tipos.

Do total de referências bibliográficas citadas, apresenta-se em destaque a referência a livros, com $64,4 \%$ desse total, seguida da referência a periódicos, com $18,8 \%$. Com relação a este aspecto, deve-se ter cuidado na análise dos dados relativos a 1998 e 1999, visto que se tem apenas um único trabalho para cada ano, o que limita as evidências deste período. A Tabela 11 resume os resultados da tabulação dos dados sobre referências bibliográficas:

Tabela 11: Tipos de Bibliografia Referenciada

\begin{tabular}{|c|c|c|c|c|c|}
\hline \multicolumn{2}{|c|}{ Tipo de Bibliografia } & Total por Tipo & $\%$ & Total Geral & $\%$ \\
\hline \multirow{2}{*}{ Livros } & Nacional & 127 & $24,4 \%$ & \multirow{2}{*}{335} & \multirow{2}{*}{$64,4 \%$} \\
\hline & Internacional & 208 & $40,0 \%$ & & \\
\hline \multirow{2}{*}{ Teses e Dissertações } & Nacional & 35 & $6,7 \%$ & \multirow{2}{*}{35} & \multirow{2}{*}{$6,7 \%$} \\
\hline & Internacional & - & $0,0 \%$ & & \\
\hline \multirow{2}{*}{ Periódicos } & Nacional & 27 & $5,2 \%$ & \multirow{2}{*}{98} & \multirow{2}{*}{$18,8 \%$} \\
\hline & Internacional & 71 & $13,7 \%$ & & \\
\hline \multirow{2}{*}{ Anais Eventos Científicos } & Nacional & 23 & $4,4 \%$ & \multirow{2}{*}{33} & \multirow{2}{*}{$6,3 \%$} \\
\hline & Internacional & 10 & $1,9 \%$ & & \\
\hline \multirow{2}{*}{ Sites Web } & Nacional & 6 & $1,2 \%$ & \multirow{2}{*}{6} & \multirow{2}{*}{$1,2 \%$} \\
\hline & Internacional & - & $0,0 \%$ & & \\
\hline \multirow{2}{*}{ Jornais e Magazines } & Nacional & 2 & $0,4 \%$ & \multirow{2}{*}{2} & \multirow{2}{*}{$0,4 \%$} \\
\hline & Internacional & - & $0,0 \%$ & & \\
\hline \multirow{2}{*}{ Normas } & Nacional & - & $0,0 \%$ & \multirow{2}{*}{1} & \multirow{2}{*}{$0,2 \%$} \\
\hline & Internacional & 1 & $0,2 \%$ & & \\
\hline \multirow{2}{*}{ Outros tipos } & Nacional & 9 & $1,7 \%$ & \multirow{2}{*}{10} & \multirow{2}{*}{$1,9 \%$} \\
\hline & Internacional & 1 & $0,2 \%$ & & \\
\hline Total & & 520 & $100,0 \%$ & 520 & $100,0 \%$ \\
\hline
\end{tabular}

A evolução anual das citações referenciadas nos trabalhos pode ser analisada na Tabela 12, que aponta crescente utilização de livros e periódicos internacionais como fontes de consulta, superando o número de referências a obras nacionais, nos períodos de 2001 a 2003. 
Tabela 12: Evolução do Tipo de Bibliografia Referenciada

\begin{tabular}{|c|c|c|c|c|c|c|c|c|}
\hline \multicolumn{2}{|c|}{ Tipo de Bibliografia } & 1998 & 1999 & 2000 & 2001 & 2002 & 2003 & Total \\
\hline \multirow{2}{*}{ Livros } & Nacional & & 8 & 31 & 41 & 28 & 19 & 127 \\
\hline & Internacional & 3 & 1 & 28 & 62 & 86 & 28 & 208 \\
\hline \multirow{2}{*}{ Teses e Dissertações } & Nacional & & & 3 & 19 & 13 & & 35 \\
\hline & Internacional & & & & & & & \\
\hline \multirow{2}{*}{ Periódicos } & Nacional & & 2 & 4 & 9 & 6 & 6 & 27 \\
\hline & Internacional & 4 & 1 & 8 & 2 & 22 & 34 & 71 \\
\hline Anais Eventos & Nacional & 2 & & 8 & 4 & 5 & 4 & 23 \\
\hline Científicos & Internacional & & 2 & 3 & & 3 & 2 & 10 \\
\hline \multirow{2}{*}{ Sites Web } & Nacional & & & & & 4 & 2 & 6 \\
\hline & Internacional & & & & & & & \\
\hline \multirow{2}{*}{ Jornais e Magazines } & Nacional & & & & 1 & & 1 & 2 \\
\hline & Internacional & & & & & & & \\
\hline \multirow{2}{*}{ Normas } & Nacional & & & & & & & \\
\hline & Internacional & & & & & 1 & & 1 \\
\hline \multirow{2}{*}{ Outros tipos } & Nacional & & & 2 & & 1 & 6 & 9 \\
\hline & Internacional & & & & & 1 & & 1 \\
\hline \multirow{3}{*}{ Total } & Nacional & 2 & 10 & 48 & 74 & 57 & 38 & 229 \\
\hline & Internacional & 7 & 4 & 39 & 64 & 113 & 64 & 291 \\
\hline & Geral & 9 & 14 & 87 & 138 & 170 & 102 & 520 \\
\hline \multirow{3}{*}{ Média por Trabalho } & Nacional & 2,0 & 10,0 & 9,6 & 7,4 & 6,3 & 6,3 & 7,2 \\
\hline & Internacional & 7,0 & 4,0 & 7,8 & 6,4 & 12,6 & 10,7 & 9,1 \\
\hline & Geral & 9 & 14 & 17,4 & 13,8 & 18,9 & 17,0 & 16,3 \\
\hline
\end{tabular}

Considerando o total de 520 citações no universo de 32 artigos, o número médio de referências bibliográficas foi 16,3 por artigo, das quais $44 \%$ foram nacionais e $56 \%$, internacionais.

\section{Considerações Finais}

O panorama revelado por esta pesquisa, considerando a dimensão quantitativa e qualitativa dos trabalhos apresentados na área de custos do EnANPAD, é positivo em alguns aspectos e negativo em outros. De modo geral, destaca-se o crescimento quantitativo da apresentação de trabalhos na área de contabilidade do EnANPAD. Em 1998, o primeiro ano de existência da temática no evento, o volume de trabalhos apresentados na área de contabilidade correspondeu a 4,8\% do total dos trabalhos do evento e, em 2003, a participação dessa área evoluiu para 8,89\%. 
A produção científica por autor e por região reflete a expansão dos programas de pós-graduação em contabilidade e as suas respectivas contribuições nos últimos três anos para o crescimento da área. Também merece destaque a abordagem empírica revelada em grande parte dos trabalhos apresentados, sendo esta um indicador positivo da evolução do tipo de método de pesquisa utilizado, uma vez que os resultados desses estudos não estão fundamentados exclusivamente em revisão de literatura.

O desafio permanece no que diz respeito ao rigor metodológico, destacando-se, numa abordagem positivista, o pouco uso de técnicas de modelagem para demonstrar aplicações de técnicas de custos, além da quase inexistência do emprego de métodos quantitativos em apoio de estudos empíricos.

No que diz respeito ao aspecto qualitativo do método de pesquisa empregado, os resultados obtidos sugerem falta de rigor no processo de estruturação de questionários, bem como falhas metodológicas na aplicação de técnicas de entrevistas. A qualidade da contribuição das pesquisas, baseadas em estudos de caso, também pode ser questionada, uma vez que vários trabalhos (sete), que aplicam o método de pesquisa baseado em estudos de casos, simplesmente ilustram a teoria consolidada sem o aprofundamento de análises e conclusões. Considerando as proposições de Yin (2001), com relação ao emprego do estudo de caso como método de pesquisa científica, além das considerações de Eisenhardt (1989), Otley e Berry (1994), Keating (1995) e Model (2005), podese questionar a eficácia da aplicação desse método na produção acadêmica de custos.

Tendo em vista as limitações deste estudo, no que se refere ao universo e à delimitação do período da pesquisa, as suas conclusões não podem ser generalizadas, mas devem ser entendidas como tendência da evolução da área. O desenvolvimento dos novos programas de mestrado e até mesmo de doutorado no país deve afetar quantitativamente e qualitativamente esse panorama apresentado.

O escopo desta pesquisa foi limitado, porém serve como referência para futuras pesquisas na área de contabilidade de custos. Nesta mesma direção, novos trabalhos de pesquisa podem ser empreendidos, tais como estudos comparativos entre a realidade brasileira e a de outros países, estudos mais aprofundados sobre os métodos de pesquisa aplicados e estudos mais amplos na área temática de contabilidade e controle gerencial.

Artigo recebido em 25.10.2004. Aprovado em 01.08.2005. 


\section{Referências Bibliográficas}

Atkinson, A. A,

Balakrishnan, R.,

Booth, P.,

Cote, J. M.,

Groot, T.,

Malmi, T., (1997).

Roberts, H.,

Uliana, E., \&

Wu, A. (1997).

New directions in management accounting research. Journal of Management Accounting Research, 9, 79-108.

Baxter, J., \&

Chua, W. F. (2003).

Alternative management accounting research: whence and whither. Accounting, Organizations and Society, 28(2), 97-113.

Beatty, J. E. (2002).

The head - collaborative frames at the academy of management. Journal of Management Inquiry, 11(3), 305-315.

Bhimani, A. (2002).

European management accounting research: traditions in the making. European Accounting Review, 11(1), 99-117.

Bricker, R. (1989).

An empirical investigation of the structure of accounting research. Journal of Accounting Research, 27(2), 246-262.

Burns, J. (2000).

The dynamics of accounting change: inter-play between new practices, routines, institutions, power and politics. Accounting, Auditing \& Accountability Journal, 13(5), 566596.

Burns, J., \&

Scapens, R. W. (2000).

Conceptualizing management accounting change: an institutional framework. Management Accounting Research, 11(1), 3-25.

Eisenhardt, K. M. (1989).

Building theories from case study research. Academy of Management Review, 14(4), 532-550.

Horngren, C. T. (1989).

Cost and management accounting: yesterday and today. Journal of Management Accounting Research, 3, 21-32.

Ittner, C. D., \&

Larcker, D. F. (2002).

Empirical managerial accounting research: are we just describing management consulting practice? European Accounting Review, 11(4), 787-794.

Kasanen, E., \&

Lukka, K. (1995).

Methodological themes: the problem of generalizability: anecdotes and evidence in accounting research. Accounting, Auditing \& Accountability Journal, 8(5), 71-91. 
Keating, P. J. (1995).

A framework for classifying and evaluating the theoretical contributions of case research in management accounting. Journal of Management Accounting Research, 7, 66-79.

Leal, R. P. C.,

Oliveira, J., \&

Soluri, A. F. (2003).

Perfil da pesquisa em finanças no Brasil. Revista de Administração de Empresa, 43(1), 91-103.

Machado-da-Silva, C. L.,

Cunha, V., \&

Amboni, N. (1990).

Organizações: o estado da arte da produção acadêmica. Anais do Encontro Nacional de Pósgraduação em Administração, Florianópolis, SC, 14.

Martins, G. (2002).

Considerações sobre os doze anos do caderno de estudos. Revista Contabilidade e Finanças - USP (30), 81-88.

Mitenko, G. R., \&

Diamond, J.M. (1994).

Publication policies and practices of major journals: a survey of editors.

Financial Practice and Education, 4(2), 88-93.

Model, S. (2005).

Triangulation between case study and survey methods in management accounting research: an assessment of validity implications. Management Accounting Research, 16(2), 231-242.
Oliveira, M. (2002).

Análise dos periódicos brasileiros de contabilidade. Revista Contabilidade \& Finanças - USP (29), 68-86.

Otchere, M. (2003).

Accounting and finance at forty: a retrospective evaluation. Accounting \& Finance, 43(2), 211-231.

Otley, D. T., \&

Berry, J. (1994).

Case study research in management accounting and control. Management Accounting Research, 5(5), 45-65.

Quintella, R. H. (2003).

Encontro nacional da ANPAD x meeting of AOM: lições, questionamentos e especulações. Revista de Administração de Empresa, 43(2), 107-115.

Riccio, E. L.,

Sakata, M. C. G., \&

Carastan, J. (1999, setembro/dezembro).

Accounting research in brazilian universities: 1962-1999. Caderno de estudos - FIPECAFI (22), 35-44.

Riccio, E. L.,

Sakata, M. C. G., \&

Segura, L. C. (2001).

A pesquisa qualitativa em contabilidade. Procedings of AsianPacific Conference on International Accounting Issues, Rio de Janeiro, RJ, Brasil, 13.

Scapens, R. W. (1994).

Never mind the gap: towards an institutional perspective on management accounting practice. Management Accounting Research, 5(3-4), 301-321. 
Scapens, R. W., \&

Roberts, J. (1993).

Accounting and control. a case study of resistance to accounting change. Management Accounting Research, 4(1), 1-32.

Shields, M. (1997).

Research in management accounting by north americans in the 1990s. Journal of Management Accounting Research (9), 3-60.

Sprinkle, G. B. (2003).

Perspectives on experimental research in managerial accounting Accounting, Organizations and Society, 28(2), 287-303.

\section{Sue, L. (1996).}

Theories for theorists or theories for practice? Liberating academic accounting research? Accounting, Auditing \& Accountability Journal, 9(4), 112-120.

Tonelli, M. J.,

Caldas, M. P. C.,

Lacombe, B. M. B., \&

Tinoco, T. (2003).

Produção acadêmica em recursos humanos no Brasil: 1991-2000. Revista de Administração de Empresa, 43(1), 104-122.
Vergara, S. C., \&

Carvalho Jr., D. S. (1995).

Nacionalidade dos autores referenciados na literatura brasileira sobre organizações. Anais do Encontro Nacional dos Programas de PósGraduação em Administração, Angra dos Reis, RJ, 19.

Vieira, F. G. D. (1998).

Por quem os sinos dobram? Uma análise da publicação científica na área de marketing do EnANPAD. Anais do Encontro Nacional de Programas de Pós-graduação em Administração, Foz do Iguaçu, PR, 22.

Vollmers, G. L. (1996).

Academic cost accounting from 19201950: alive and well. Journal of Management Accounting Research, 8, 183-199.

\section{Zeff, S. A. (1996).}

A study of academic research journals in accounting. Accounting Horizons, 10(3), 158-177.

Yin, R. K. (2001).

Estudo de caso: planejamento e métodos (2a ed.). (D. Grassi, Trad.). Porto Alegre: Bookman. (obra original publicada 1984). 